\title{
Impact of Nursing Staffing on Patient Outcomes in Intensive Care Unit
}

\section{David A Thompson ${ }^{1-3 *}$, Yea-Jen Hsu², Bickey H Chang ${ }^{1}$ and Jill A Marsteller ${ }^{1,2}$}

${ }^{1}$ Department of Anesthesiology and Critical Care Medicine, School of Medicine, Johns Hopkins University, Baltimore, USA

${ }^{2}$ Department of Health Policy and Management, Bloomberg School of Public Health, Johns Hopkins University, Baltimore, Maryland, USA

${ }^{3}$ Division of Acute and Chronic Care, School of Nursing, Johns Hopkins University, Baltimore, Maryland, USA

\begin{abstract}
Background: The impact of nursing care on patient outcomes is not well understood. The objective of this study is to assess the effects of nursing care hours per patient day, nursing skill mix, and nurse turnover on central line-associated bloodstream infection (CLABSI) rates, length of stay (LOS), and mortality in the context of intensive care units (ICUs) using interventions to reduce bloodstream infections and improve patient safety, teamwork and interdisciplinary communication.
\end{abstract}

Methods: This study uses longitudinal data from 45 ICUs from 35 hospitals in two faith-based health systems across 12 states. The 45 ICUs were divided into two groups. We analyzed 19 months of experience for the first group and a year of experience for the second group. We collected hours worked by all bedside nurses to calculate National Quality Forum (NQF) nursing measures. We used a two-level random-intercept model to account for the correlations among repeated measures for the same ICU. Regressions accounted for the influences of number of Intensivists, type of ICUs, ICU bed size, which health system the unit belonged to, nursing practice environment (PES-NWI) and project phase. Models also adjusted for average patient charges, to partially account for ICU casemix.

Results: Nursing care hours per patient day greater than 20 hours was associated with lower CLABSI rates. Increasing nursing hours per patient day was also associated with shorter LOS, with the strongest relationship where nursing hours per patient day was lower ( $<20$ hours compared to $\geq 20$ hours). A Higher skill mix was associated with shorter LOS but higher CLABSI rates. We found no significant relationships of nursing turnover with any outcome, or between any nursing variables and mortality.

Conclusions: Our findings suggest that nursing care hours per patient day and nursing skill mix significantly contribute to CLABSI prevention and LOS in the ICU setting.

Keywords: Nurse staffing; Nurse turnover; Patient safety; Intensive care

\section{Introduction}

The landmark report, Crossing the Quality Chasm: A New Health System for the $21^{\text {st }}$ Century highlighted the difference between the quality of care that is, and the quality of care that should be, provided in the United States [1]. In the many years since the publication of this report, clinicians and researchers have struggled to achieve what is best for our patients while allowing them to be involved in their care. However, there is much that we still do not fully understand about optimizing patient care and patient outcomes. One of these important areas is the impact of nurses on patient outcomes. Although nurses make up one of the largest clinician populations, nurses have said for years that there are simply not enough of them to provide quality patient care $[2,3]$. Nurses have long felt that they are underappreciated and face unrealistic workloads $[3,4]$. Highlighting the importance of the nurse role in health care delivery, in 2009 the National Quality Forum (NQF) endorsed several nurse-staffing related quality indicators developed by the American Nurses Association [5].

The Institute of Medicine (IOM) committee's Nursing Staff in Hospitals and Nursing Homes highlighted a major gap in research linking nursing staffing to the quality of patient care [6]. Research in this area began mostly as part of studies focusing on the effect of other organizational characteristics [7-11]. More recently, studies specifically focused on examining the relationship between nursing staffing and the patient outcome have increased markedly in number [4,12-17]. Systematic reviews and recent studies suggest that higher levels of nurse staffing, usually measured as nursing hours per patient day or nurse-to-patient ratio, may be associated with improved patient outcomes, including lower hospital mortality, failure to rescue, and adverse events, but studies continue to report mixed results [18-22]. Inconsistencies in data sources, measurement of nurse staffing, and adjustment methodologies for confounders have made it difficult to explain the mixed results and to make definitive statements about how nursing staffing affects patient outcomes. Studies with large sample size tend to obtain nursing staffing data from large administrative databases, which may introduce bias. As an example, many studies use American Hospital Association (AHA) annual survey data, which do not distinguish between inpatient and outpatient staffing and lead to attenuation bias [23]. Some studies use hospital payroll records, which usually do not include contract nurses [24]. On the other hand, studies which directly measure nurse staffing, accounting for only hours of direct patient care, tend to have other limitations, in that they are usually conducted in single hospital or state settings, or are crosssectional in nature $[4,12,15,20]$.

Further, most studies in the past 10 years have focused on outcomes at a general inpatient level or aggregated at the hospital level and provided little guidance on staffing levels for intensive care units (ICUs) $[20,25]$. Reviews of the smaller subset of studies focused on ICU settings show similar methodological inconsistencies and inconclusive results [25-27]. To further knowledge on this topic, there is need for large, prospective, longitudinal, multi-center trials which use standardized nurse staffing and outcome measure definitions $[18,25]$.

*Corresponding author: David A Thompson, Johns Hopkins University School of Medicine, Anesthesiology and Critical Care Medicine, Armstrong Institute for Patient Safety and Quality, 750 East Pratt Street, 15th Floor (room 1525) Baltimore, MD 21202, USA, Tel: 410-637-4392; Fax: 410-502-3235; E-mail: dthomps1@jhmi.edu

Received May 07, 2013; Accepted June 25, 2013; Published July 01, 2013 Citation: Thompson DA, Hsu YJ, Chang BH, Marsteller JA (2013) Impact of Nursing Staffing on Patient Outcomes in Intensive Care Unit. J Nurs Care 2: 128 doi:10.4172/2167-1168.1000128

Copyright: (c) 2013 Thompson DA, et al. This is an open-access article distributed under the terms of the Creative Commons Attribution License, which permits unrestricted use, distribution, and reproduction in any medium, provided the original author and source are credited. 
In this study we hypothesized that nursing measures would be correlated with a reduction in central line-associated bloodstream infection (CLABSI) rates, length of stay (LOS), and mortality in ICUs. The Robert Wood Johnson Foundation's Interdisciplinary Nursing Quality Research Initiative (INQRI) provided us an opportunity to assess the National Quality Foundation (NQF) nursing quality measures against patient outcomes as part of a larger study testing a nurse-led CLABSI reduction intervention in a multisite, phased, cluster-randomized controlled trial [28].

\section{Methods}

Based on a framework adapted from Donabedian's structureprocess-outcome approach [29], we hypothesized that nursing skill mix (a structural factor), nursing hours per patient care (a process factor), and nurse turnover (which can be considered both a process and an outcome variable)would be associated with patient outcomes in the ICU (Figure 1). Other structural variables (e.g., bed size, ICU type) and process variables (e.g., program interventions implemented, activities of quality improvement teams) were expected to influence outcomes as well. Moreover, the culture of patient safety is the overarching context of care variable which is related to all three Donabedian levels. The safety culture can be accessed through climate variables such as teamwork climate and Nursing Practice Environment [30,31]. Based on this framework, the researchers developed the plans and instruments for data collection and analysis.

\section{Data Source}

Data for this study were drawn from a cluster randomized controlled trial aimed to promote a culture of patient safety and to reduce CLABSI rates [28]. The 45 participating ICUs were from 35 faith-based not-forprofit community hospitals in two affiliated systems located in 12 states. Forty-five ICUs were randomized into two intervention groups with the first group having 23 ICUs and the second 22 ICUs. The first group started the implementation of the interventions in March 2007 and the second group began 7 months later in October 2007. The project ended in September 2008. Details regarding the trial and the interventions are provided elsewhere [28]. The study was approved by the institutional review board of Johns Hopkins University School of Medicine.

Nurse Managers in each ICU reported unit-level data on nurse staffing and nurse turnover on a monthly basis. The infection preventionists at each hospital collected number of CLABSIs and central line days according to the definitions of the Centers for Disease Control and Prevention (CDC). Other outcomes were extracted from administrative data by staff in the health system's corporate headquarters, which forwarded all of the data, including nurse staffing, patient outcomes, and ICU and hospital characteristics, to the research

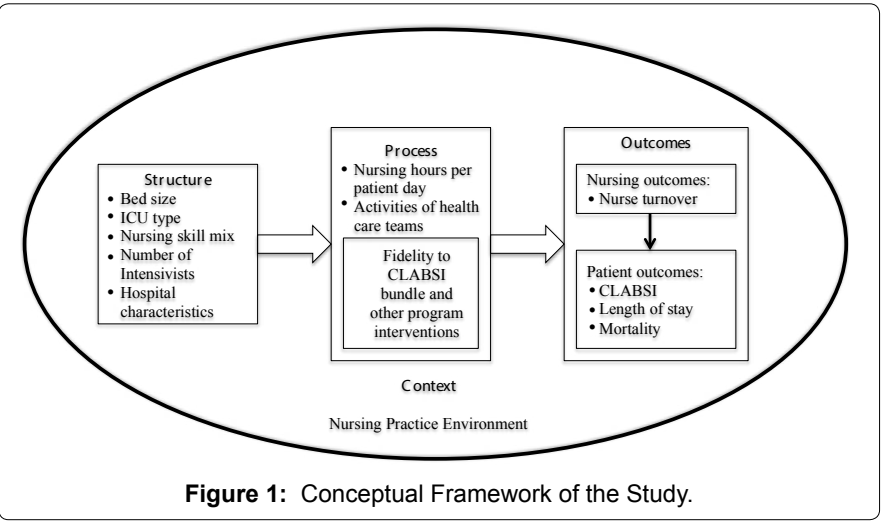

team. Units sent baseline data from 2006 directly to the research team based on their own. All patient outcome variables in this study were $100 \%$ complete. The missing rate of nurse staffing variables is $3.3 \%$ at the ICU-month level.

At the beginning of the program, the Practice Environment Scale of the Nursing Workforce Index (PES-NWI) was administered to ICU nurses regardless of their involvement in the intervention. All nurses with a $50 \%$ or greater commitment to the ICU for at least the 4 consecutive weeks prior to survey administration were requested to complete the PES-NWI. We surveyed all nurses rather than a partial sample to reduce the risk of random error in the assessment of context of care. The average response rate across the participating units was $76 \%$.

\section{Measures of staffing, skill mix, and turnover}

We used the National Quality Forum (NQF) nursing measures for this study [5]. We collected data on the number of registered nurses (RNs) and unlicensed assistive personnel (UAP) using full-timeequivalents (FTEs) on the last day of each month, the number of RN FTEs that resigned or transferred out of the unit for the month, and also unit-level number of patient days for the month. The level of nurse staffing was estimated by hours. We calculated monthly total nursing care hours by multiplying the number of FTEs on the last day of the month by $1731 / 3$ hours (the number of work hours in a month assuming a standard year of 2080 hours, or 52 weeks at 40 hours per week).

\section{Overview of NQF Measures}

Nursing care hours per patient day: We calculated this measure for all nursing staff, including RNs (licensed practical nurses are not included in the staff of Adventist ICU hospitals) and unlicensed assistive personnel (UAP). The numerator is the number of productive hours worked by RNs and UAP with direct patient care responsibilities. This includes all bedside nurses, regardless of their affiliation as full time or part time active staff, agency or other contractual nurses. The denominator is all patient days for the unit.

Nursing skill mix: The numerator is defined as the number of productive hours worked by all nursing staff with bedside or patient care responsibilities. Again this includes any RN workforce and any UAP. This is divided by the denominator which is the total number of productive hours worked by the RN staff with direct care responsibilities. The high values of this measure indicate larger proportion of UAP staff hours relative to $\mathrm{RN}$ hours. The minimum skill mix measure of 100 represents an exclusively RN staff in the unit with no help from UAP; higher values of skill mix indicate rising UAP hours relative to RN hours.

Nursing turnover: The nurse manager of each participating ICU kept and reported the number of nurses who transfer from the ICU to another inpatient unit within the hospital, defined as internal turnover. They also collected data on external turnover, the number of nurses who voluntarily leave the ICU to work in another hospital. The two determine the numerator for the measure. The denominator is the number of both full and part time nurses on the last day of the month. Both numerator and denominator are measured using FTEs. Nurses that are excluded from the sample include those absent because of death, illness, retirement, disciplinary action and permanent reductions in staff.

\section{Measure of practice environment}

The Practice Environment Scale of the Nursing Workforce Index (PES-NWI) is a validated tool designed to contain all factors associated 
with job satisfaction and the quality of nursing care delivery [30]. The instrument consists of 31 items on a 4-point Likert scale measuring 5 organizational traits common in nursing-magnet hospitals: nursing participation in hospital affairs; nursing foundations for quality of care; nurse manager ability, leadership, and support of nurses; staffing and resource adequacy; and the degree of collegial nurse/physician relationships. The composite score, which is a mean of the 5 subscale scores, was used in this study to adjust for context differences.

\section{Measures of patient outcomes}

We analyzed three measures of patient outcomes, including CLABSI rates, ICU length of stay (LOS), and in-ICU mortality. Primary CLABSIs were determined using the following criteria: bloodstream infections in ICU patients aged 18 years and older with a laboratory confirmed CLABSI who had central lines in place within the 48-hour period before the development of the infection. Non-ICU patients, patients without central lines, secondary bloodstream infections, and those present or incubating within 72 hours of admission to the unit were excluded. We calculated the monthly rate of CLABSI by dividing the number of infections by the number of central line days and expressed it as the number of CLABSIs per 1000 line days. We calculated mortality rates by dividing the number of in-ICU deaths by the total number of patients admitted to the ICU for the month and expressed them as the number of deaths per 100 cases. ICU average LOS was calculated by dividing total patient days for the month by the total number of patients admitted to the ICU for the month. Table 1 provides the formulae for the key measures described above.

\section{Statistical analysis}

The unit of analysis was the ICU-month. We analyzed 19 months of experience for the first intervention group and a year of experience for the second intervention group. For each outcome, we conducted bivariate and multivariate regression analyses with the nursing measures and control variables. In all multivariate regression analyses, we controlled for practice environment scale, structural variables (ICU type, ICU bed size, number of Intensivists in the ICU, number of ICUs in the hospital, and the health system the hospital belonged to), and a set of dummy variables for the phase of the intervention to account for any impact of a time trend and the intervention used in the program. In the regression on mortality we also included average monthly charges per patient to partially control for casemix.

CLABSIs are measured as a count with a high proportion of zeroes ( $87 \%$ of values) and over dispersion [32]. To address the nature of

\begin{tabular}{|l|l|}
\hline Variables & \\
\hline $\begin{array}{l}\text { Nurse staffing and turnover measures } \\
\text { Nursing care } \\
\text { hours per } \\
\text { patient day }\end{array}$ & $\frac{(\text { RNFTEs }+ \text { UAP FTEs }) \times 2080 \div 12}{\text { unit patient days }}$ \\
\hline Skill mix & $\frac{(\text { RNFTEs }+ \text { UAP FTEs })}{\text { RN FTEs }} \times 100$ \\
\hline Nurse turnover & $\frac{(\text { RNFTEs resigned or transferred + UAP FTEs resigned or transferred })}{\text { RNFTEs end of month + UAP FTEs end of month }} \times 100 \%$ \\
\hline Patient outcome & measures \\
\hline CLABSI rate & $\frac{\text { number of CLABSIs }}{\text { number of central line days }} \times 1000$ \\
\hline LOS & $\frac{\text { unit patient days }}{\text { number of patients admitted }}$ \\
\hline $\begin{array}{l}\text { In-ICU } \\
\text { mortality }\end{array}$ & $\frac{\text { number of deaths }}{\text { number of patients admitted }} \times 100$ \\
\hline
\end{tabular}

Table 1: Operational Definitions for Primary Variables.

\begin{tabular}{|c|c|c|}
\hline Characteristics & $n=45$ & $\%$ \\
\hline \multicolumn{3}{|l|}{ Type of ICUs* } \\
\hline Coronary/Cardiovascular & 8 & 17.8 \\
\hline Medical & 1 & 2.2 \\
\hline Mixed & 34 & 75.6 \\
\hline Neurosurgical & 1 & 2.2 \\
\hline Surgery & 1 & 2.2 \\
\hline \multicolumn{3}{|l|}{ System } \\
\hline East & 35 & 77.8 \\
\hline West & 10 & 22.2 \\
\hline No. of ICU beds (median, IQR) & 10 & $(8-18)$ \\
\hline No. of registered nurses (median, IQR) & 25 & $(19-45)$ \\
\hline No. of Intensivists (median, IQR) & 0 & $(0-2)$ \\
\hline PES-NWI (median, IQR) & 2.83 & $(2.63-2.98)$ \\
\hline \multicolumn{3}{|c|}{ Nurse staffing at baseline, year 2006 (median, IQR) } \\
\hline Nursing care hours per patient day & 17.0 & $(15.2-20.1)$ \\
\hline Nursing skill mix & 104.5 & $(100.0-111.0)$ \\
\hline Nurse turnover & 13.6 & $(7.2-19.4)$ \\
\hline \multicolumn{3}{|c|}{ Patient outcome variables at baseline, year 2006 (median, IQR) } \\
\hline No. of CLABSIs per 1000 central line days & 1.89 & $(0.72-4.18)$ \\
\hline Length of stay & 3.44 & $(3.05-3.78)$ \\
\hline No. of deaths per 100 cases & 6.63 & $(5.23-8.10)$ \\
\hline
\end{tabular}

ICU: intensive care unit; CLABSI: central line-associated bloodstream infection; PES-NWI: Practice Environment Scale of the Nursing Work Index

Table 2: Baseline Characteristics of Participating ICUs.

the CLABSI as a dependent variable, we used a zero-inflated negative binomial (ZINB) model, which is a special mixture model with two classes, where the first class has a fixed value at 0 and might include observations with very low risk of CLABSI, due to a well-structured context or system. The second class uses a negative binomial model to specify the other zeroes and the continuous positive portion of the distribution. We adjusted for a zero rate for year 2006, ICU type, ICU bed size, number of ICUs in the hospital, the health system the hospital belonged to, and project phase, to account for the very low risk of CLABSI. An offset for the number of central line days adjusted for the risk of infection faced at each ICU-month. We also used robust clustering to account for shared variation among monthly observations from the same ICU. Nursing care hours per patient day was modeled as a binary variable in the regression indicating nursing care hours less than 20 hours or 20 hours and greater, based on the crude relationship observed. Nursing skill mix and nurse turnover were modeled as continuous variables.

We used two-level random intercept models to analyze the impact of nurse staffing and turnover on LOS and in-ICU mortality. In these models, we used unit-level random intercepts to account for the clustering among repeated measures for the same unit. Regressions also adjusted for average charges per patient admitted in the month to partially control for ICU casemix. We used a normal linear distribution to analyze LOS, and a Poisson distribution with an offset for the number of admitted patients in the unit to analyze mortality. A spline approach was applied to model the nursing care hours per patient day, with a cut point of 20 hours, to predict LOS.

All analyses were conducted using STATA version 12.1 (Stata Corporation, College Station, Texas).

\section{Results}

Table 2 provides participating ICUs' key characteristics and 
Citation: Thompson DA, Hsu YJ, Chang BH, Marsteller JA (2013) Impact of Nursing Staffing on Patient Outcomes in Intensive Care Unit. J Nurs Care 2: 128. doi:10.4172/2167-1168.1000128

Page 4 of 6

summary statistics for the nurse staffing, turnover, and patient outcomes in year 2006 before the units joined the study. The majority of these ICUs were of mixed specialty (76\%) and $18 \%$ were coronary/cardiovascular ICUs. The bed size of these ICUs ranged from 4 to 32 with a median of 10 beds. Sixty-nine percent of them had no Intensivists in the unit. In 2006, the participating ICUs had median nursing care hours per patient day of 17 . The median UAP staff hours were about $5 \%$ of the RN staff hours. The median annual nurse turnover rate was $14 \%$. The median CLABSI rate and in-ICU mortality were 1.9 CLABSIs per 1000 central line days and 6.6 deaths per 100 cases, respectively. The median LOS across ICUs was 3.4 days.

Tables 3-5 indicate that nursing care hours per patient day is one of the most influential explanatory variables in both bivariate and multivariate regressions. Controlling for other influences, nursing care hours per patient day greater than 20 was associated with a near $60 \%$ reduction in the CLABSI rate compared to ICU-months with nursing care hours per patient day less than 20 (IRR $=0.44 ; 95 \% \mathrm{CI}=0.25-0.77$; $\mathrm{p}=0.004)$. A higher nursing care hour per patient day was also associated with shorter LOS, and the association was stronger when nursing hours per patient day was less than 20 . When we modeled nursing care hours per patient day using a spline approach, a one-hour increase in nursing care hours per patient day was associated with a four-fold reduction

\begin{tabular}{|c|c|c|c|c|c|c|}
\hline \multirow[b]{2}{*}{$\begin{array}{l}\text { Negative binomial } \\
\text { part }\end{array}$} & \multicolumn{3}{|c|}{ Bivariate } & \multicolumn{3}{|c|}{ Multivariate } \\
\hline & IRR & $95 \% \mathrm{Cl}$ & $P$ Value & IRR & $95 \% \mathrm{Cl}$ & $\begin{array}{l}P \\
\text { Value }\end{array}$ \\
\hline \multicolumn{7}{|c|}{$\begin{array}{l}\text { Nursing care hours per } \\
\text { patient day }\end{array}$} \\
\hline$<20$ & 1.00 & & & 1.00 & & \\
\hline$\geq 20$ & 0.45 & $(0.25-0.81)$ & 0.008 & 0.44 & $(0.25-0.77)$ & 0.004 \\
\hline Nursing skill mix & 0.96 & $(0.94-0.98)$ & $<0.001$ & 0.96 & $(0.94-0.99)$ & 0.009 \\
\hline Nurse turnover & 1.02 & $(0.96-1.09)$ & 0.433 & 1.00 & $(0.94-1.06)$ & 0.991 \\
\hline No. of Intensivists & 0.93 & $(0.79-1.10)$ & 0.413 & 0.99 & $(0.84-1.15)$ & 0.863 \\
\hline PES-NWI & 0.61 & $(0.18-2.03)$ & 0.419 & 1.26 & $(0.39-4.12)$ & 0.698 \\
\hline \multicolumn{7}{|l|}{ ICU type } \\
\hline Mixed & 1.00 & & & 1.00 & & \\
\hline Coronary & 1.10 & $(0.30-4.06)$ & 0.882 & 0.45 & $(0.21-0.93)$ & 0.032 \\
\hline Other & 1.01 & $(0.30-3.44)$ & 0.990 & 0.33 & $(0.12-0.87)$ & 0.025 \\
\hline \multicolumn{7}{|l|}{ No. of ICU beds } \\
\hline $1-10$ & 1.00 & & & 1.00 & & \\
\hline $11-20$ & 0.43 & $(0.15-1.28)$ & 0.131 & 0.71 & $(0.46-1.10)$ & 0.127 \\
\hline$\geq 21$ & 0.66 & $(0.22-2.01)$ & 0.468 & 0.90 & $(0.27-2.99)$ & 0.863 \\
\hline \multicolumn{7}{|l|}{$\begin{array}{l}\text { No. of ICUs in the } \\
\text { hospital }\end{array}$} \\
\hline One & 1.00 & & & 1.00 & & \\
\hline Two & 1.03 & $(0.41-2.56)$ & 0.949 & 1.47 & $(0.52-4.16)$ & 0.471 \\
\hline More than four & 0.66 & $(0.23-1.89)$ & 0.439 & 0.67 & $(0.31-1.42)$ & 0.296 \\
\hline \multicolumn{7}{|l|}{ System } \\
\hline East & 1.00 & & & 1.00 & & \\
\hline West & 1.56 & $(0.81-3.00)$ & 0.179 & 1.24 & $(0.63-2.43)$ & 0.532 \\
\hline \multicolumn{7}{|l|}{ Intervention phase } \\
\hline $1-3 \mathrm{mo}$ & 1.00 & & & 1.00 & & \\
\hline 4-6 mo & 1.00 & $(0.36-2.76)$ & 0.999 & 1.27 & $(0.62-2.62)$ & 0.511 \\
\hline 7-9 mo & 1.73 & $(0.71-4.23)$ & 0.226 & 2.42 & $(1.26-4.66)$ & 0.008 \\
\hline $10-12 \mathrm{mo}$ & 1.36 & $(0.38-4.83)$ & 0.632 & 0.92 & $(0.31-2.71)$ & 0.884 \\
\hline $13-15 \mathrm{mo}$ & 1.43 & $(0.02-124.05)$ & 0.875 & 1.23 & $(0.15-10.39)$ & 0.847 \\
\hline$\geq 15 \mathrm{mo}$ & 0.94 & $(0.25-3.59)$ & 0.933 & 1.49 & $(0.42-5.27)$ & 0.535 \\
\hline
\end{tabular}

CLABSI: Central Line-Associated Bloodstream Infection; ICU: Intensive Care Unit; PES-NWI: Practice Environment Scale of the Nursing Work Index

Table 3: Regressions of Nurse Staffing and Turnover on CLABSI rates.

\begin{tabular}{|c|c|c|c|c|c|c|}
\hline & \multicolumn{3}{|c|}{ Bivariate } & \multicolumn{3}{|c|}{ Multivariate } \\
\hline & Coef. & S.E. & $P$ Value & Coef. & S.E. & $P$ Value \\
\hline \multicolumn{7}{|l|}{$\begin{array}{l}\text { Nursing care hours per patient } \\
\text { day }\end{array}$} \\
\hline$<20$ & -0.085 & 0.009 & $<0.001$ & -0.046 & 0.006 & $<0.001$ \\
\hline$\geq 20$ & -0.020 & 0.003 & $<0.001$ & -0.012 & 0.002 & $<0.001$ \\
\hline Nursing skill mix & 0.003 & 0.003 & 0.281 & 0.006 & 0.002 & 0.002 \\
\hline Nurse turnover & -0.003 & 0.005 & 0.621 & -0.003 & 0.003 & 0.421 \\
\hline No. of Intensivists & -0.016 & 0.039 & 0.673 & -0.043 & 0.053 & 0.418 \\
\hline $\begin{array}{l}\text { Average charge per case (in } \\
\text { thousands) }\end{array}$ & 0.046 & 0.002 & $<0.001$ & 0.045 & 0.002 & $<0.001$ \\
\hline PES-NWI & 0.155 & 0.391 & 0.692 & -0.691 & 0.387 & 0.074 \\
\hline \multicolumn{7}{|l|}{ ICU type } \\
\hline Mixed & - & & & - & & \\
\hline Coronary & 0.326 & 0.224 & 0.146 & -0.253 & 0.298 & 0.396 \\
\hline Other & 0.045 & 0.343 & 0.895 & -0.532 & 0.478 & 0.265 \\
\hline \multicolumn{7}{|l|}{ No. of ICU beds } \\
\hline $1-10$ & - & & & - & & \\
\hline $11-20$ & 0.193 & 0.195 & 0.321 & -0.434 & 0.204 & 0.033 \\
\hline$\geq 21$ & 0.349 & 0.231 & 0.130 & -0.521 & 0.321 & 0.104 \\
\hline \multicolumn{7}{|l|}{ No. of ICUs in the hospital } \\
\hline One & - & & & - & & \\
\hline Two & 0.169 & 0.248 & 0.495 & -1.150 & 0.366 & 0.002 \\
\hline More than four & 0.460 & 0.211 & 0.029 & -0.678 & 0.296 & 0.022 \\
\hline \multicolumn{7}{|l|}{ System } \\
\hline East & - & & & - & & \\
\hline West & 0.048 & 0.209 & 0.819 & -1.151 & 0.212 & $<0.001$ \\
\hline \multicolumn{7}{|l|}{ Intervention phase } \\
\hline $1-3 \mathrm{mo}$ & - & & & - & & \\
\hline 4-6 mo & 0.017 & 0.060 & 0.777 & -0.043 & 0.037 & 0.253 \\
\hline $7-9 \mathrm{mo}$ & 0.088 & 0.060 & 0.142 & -0.045 & 0.038 & 0.228 \\
\hline $10-12 \mathrm{mo}$ & 0.011 & 0.060 & 0.855 & -0.115 & 0.038 & 0.003 \\
\hline $13-15 \mathrm{mo}$ & 0.084 & 0.076 & 0.267 & -0.219 & 0.049 & $<0.001$ \\
\hline$\geq 15 \mathrm{mo}$ & -0.048 & 0.070 & 0.496 & -0.291 & 0.045 & $<0.001$ \\
\hline
\end{tabular}

LOS: length of stay; ICU: intensive care unit; PES-NWI: Practice Environment Scale of the Nursing Work Index

Table 4: Regressions of Nurse Staffing and Turnover on LOS.

in LOS when the hours per patient day was less than 20 (coef.=-0.05; $\mathrm{p}<0.001$ ), compared with the reduction in LOS when the hours per patient day was higher than 20 (coef. $=-0.01 ; \mathrm{p}<0.001$ ). The difference in the magnitude of the association between less than 20 and 20 or more nursing care hours per patient day was statistically significant $(\mathrm{p}<0.001)$

For skill mix, a higher proportion of UAP hours was associated with slightly longer LOS (coef. $=0.006 ; \mathrm{p}=0.002$ ) and somewhat lower CLABSI rates (IRR $=0.96 ; 95 \% \mathrm{CI}=0.94-0.99 ; \mathrm{p}=0.009$ ), other things equal. Nurse turnover was not found to be associated with any of the patient outcomes examined. None of the nursing measures tested were significantly related to in-ICU mortality in this study.

\section{Discussion}

Recent reviews of studies examining nursing staffing and patient outcomes in the ICU context have shown mixed results [25-27]. This study seeks to extend the current literature in this area by testing the association of the NQF-endorsed nurse staffing measures on ICU outcome measures, directly matching staffing and outcome 


\begin{tabular}{|c|c|c|c|c|c|c|}
\hline & \multicolumn{3}{|c|}{ Bivariate } & \multicolumn{3}{|c|}{ Multivariate } \\
\hline & IRR & $95 \% \mathrm{Cl}$ & $P$ Value & IRR & $95 \% \mathrm{Cl}$ & $P$ Value \\
\hline $\begin{array}{l}\text { Nursing care hours per } \\
\text { patient day }\end{array}$ & 1.00 & $(0.99-1.00)$ & 0.276 & 1.00 & $(0.99-1.01)$ & 0.469 \\
\hline Nursing skill mix & 1.00 & $(1.00-1.01)$ & 0.263 & 1.01 & $(1.00-1.01)$ & 0.118 \\
\hline Nurse turnover & 1.00 & $(0.99-1.02)$ & 0.454 & 1.00 & $(0.99-1.02)$ & 0.427 \\
\hline No. of Intensivists & 1.00 & $(0.95-1.05)$ & 0.925 & 1.02 & $(0.96-1.09)$ & 0.515 \\
\hline $\begin{array}{l}\text { Average charge per } \\
\text { case (in thousands) }\end{array}$ & 1.00 & $(1.00-1.01)$ & 0.051 & 1.00 & $(1.00-1.01)$ & 0.072 \\
\hline PES-NWI & 0.95 & $(0.56-1.60)$ & 0.850 & 0.68 & $(0.42-1.10)$ & 0.116 \\
\hline \multicolumn{7}{|l|}{ ICU type } \\
\hline Mixed & 1.00 & & & 1.00 & & \\
\hline Coronary & 0.76 & $(0.56-1.02)$ & 0.065 & 0.85 & $(0.60-1.19)$ & 0.341 \\
\hline Other & 0.84 & $(0.53-1.33)$ & 0.448 & 0.84 & $(0.46-1.54)$ & 0.571 \\
\hline \multicolumn{7}{|l|}{ No. of ICU beds } \\
\hline $1-10$ & 1.00 & & & 1.00 & & \\
\hline $11-20$ & 1.07 & $(0.82-1.38)$ & 0.615 & 0.99 & $(0.77-1.26)$ & 0.929 \\
\hline$\geq 21$ & 0.88 & $(0.65-1.19)$ & 0.402 & 0.64 & $(0.42-0.96)$ & 0.032 \\
\hline \multicolumn{7}{|l|}{$\begin{array}{l}\text { No. of ICUs in the } \\
\text { hospital }\end{array}$} \\
\hline One & 1.00 & & & 1.00 & & \\
\hline Two & 0.88 & $(0.63-1.23)$ & 0.440 & 0.76 & $(0.48-1.19)$ & 0.231 \\
\hline More than four & 0.80 & $(0.60-1.07)$ & 0.136 & 0.86 & $(0.60-1.22)$ & 0.388 \\
\hline \multicolumn{7}{|l|}{ System } \\
\hline East & 1.00 & & & 1.00 & & \\
\hline West & 1.48 & $(1.16-1.90)$ & 0.002 & 1.34 & $(1.02-1.76)$ & 0.037 \\
\hline \multicolumn{7}{|l|}{ Intervention phase } \\
\hline $1-3 \mathrm{mo}$ & 1.00 & & & 1.00 & & \\
\hline $4-6 \mathrm{mo}$ & 0.94 & $(0.85-1.04)$ & 0.231 & 0.93 & (0.84-1.03) & 0.183 \\
\hline 7-9 mo & 1.00 & $(0.90-1.11)$ & 0.997 & 0.98 & $(0.88-1.10)$ & 0.757 \\
\hline $10-12 \mathrm{mo}$ & 0.99 & $(0.89-1.10)$ & 0.838 & 0.98 & $(0.88-1.09)$ & 0.695 \\
\hline $13-15 \mathrm{mo}$ & 1.04 & $(0.91-1.18)$ & 0.577 & 0.99 & $(0.87-1.14)$ & 0.908 \\
\hline$\geq 15 \mathrm{mo}$ & 0.87 & $(0.77-0.99)$ & 0.034 & 0.85 & $(0.74-0.97)$ & 0.017 \\
\hline
\end{tabular}

ICU: intensive care unit; PES-NWI: Practice Environment Scale of the Nursing Work Index

Table 5: Regressions of Nurse Staffing and Turnover on Mortality.

data at the unit level. We collected the amount of time worked by all bedside nursing staff that provided direct care to patients, which is preferred to staffing measures derived from administrative databases that include RNs not involved in beside care and may introduce bias through imputation for missing data from hospital averages. The large, longitudinal, multi-center nature of the study also provides valuable insight into staffing and outcomes, adjusting for time-invariant hospital attributes that may affect both staffing and outcomes but often went unmeasured in previous studies.

This study showed that higher nursing care hours was associated with fewer CLABSIs and shorter LOS, which is consistent with trends in previous studies [33]. Moreover, the results also suggest a nursestaffing benchmark to consider. Previous studies have not been able to determine optimal nurse-to-patient ratios, and the minimum ratio implemented in California, the first state to enact a mandate on staffing ratios, was not associated with significant changes in patient outcomes $[19,34,35]$. Our results suggest that at least 20 direct nursing hours per patient day in the ICU is associated with fewer CLABSIs as compared with fewer nursing hours per patient day. Further, increasing direct nursing hours per patient day had a stronger effect on LOS in the less than 20 range. Our study and previous studies reporting nursing hours per patient day [36] suggest a shortage of nursing care in the ICU setting, since the majority of ICUs had nursing hours per patient day below this level. Additional study is needed to test this threshold, however, since nursing hours per patient day was not associated with mortality in these data.

The relationships between nursing skill mix and patient outcomes are not consistent in this study. We anticipated that lower skill mix, indicating a preponderance of RNs, would lead to shorter LOS, lower CLABSI, and reduced mortality. However, we found that higher skill mix, indicating high run licensed assistant hours, was associated with longer LOS as predicted, but also with lower CLABSI, contrary tour hypothesis. Because of the lack of LPNs in the hospitals in this dataset, middle and higher ranges of skill mix likely indicate the addition of UAPs, not the replacement of RNs by other types of nurses. These results suggest that at any given level of nursing hours per patient day, having the assistance of UAPs can lead to better care, perhaps by allowing RNs to concentrate on care provision rather than on tasks that do not require a nursing degree. Additional staff assistance may have permitted more time to implement, or better overall adoption of, the interventions used in the collaborative (i.e., CLABSI prevention bundle, checklist, daily goals sheet, etc.).

The lack of association of nurse turnover with any of these outcomes was contrary to our hypotheses. Prior literature may have used different definitions of turn over [37,38]. Further, nursing turnover does not automatically imply dilution of experience; often it may be the new nurse, fresh out of school, which leaves the ICU setting after deciding it is not his cup of tea. The lack of association of nurse staffing measures with mortality is somewhat puzzling. Previous studies show a trend of lower mortality with higher nurse staffing, although results were inconclusive $[24,25]$. It may be that there was insufficient variation in mortality to detect a relationship with nursing measures. None of the intervention periods showed significant reductions in mortality until more than 15 months post implementation; only half of the ICUs had this much experience in the study. Infections were reduced dramatically, but infections are just one type of adverse event amongst the many types of illnesses and co morbidities in the ICU patient population.

This study is subject to several limitations. First, only 45 ICUs in two faith-based healthcare systems were studied. The results may not be generalizable to all the hospitals in the US. Moreover, we did not collect patient-level data; thus, the patient casemix in the ICU was not adjusted in the models. However, in the length of stay and mortality regression, we included average charges per patient to partially account for the severity of patient status at the unit level. We also assume that patients in the ICU are generally in serious condition and the crosssectional variation in patient severity is likely to be moderate.

\section{Conclusions}

Nursing makes important contributions to health care quality and patient outcomes in ICUs. Appropriate levels of nurse staffing, particularly greater nursing hours and the availability of support staff can play key roles in preventing infections and reducing length of stay.

\section{Acknowledgements}

The authors would like to acknowledge the contributions to the larger INQR project of J Bryan Sexton, Sean Berenholtz, Janey Hsiao, Christen Fullwood, Christine Goeschel and Peter J Pronovost.

\section{References}

1. Horak BJ, Welton W, Shortell S (2004) Crossing the quality chasm: implications for health services administration education. J Health Adm Educ 21: 15-38. 
2. Aiken LH, Clarke SP, Sloane DM (2000) Hospital restructuring: does it adversely affect care and outcomes? J Nurs Adm 30: 457-465.

3. Buerhaus PI, Donelan K, Ulrich BT, Norman L, Dittus R (2006) State of the registered nurse workfore in the United States. Nurs Econ 24: 6-12, 3.

4. Aiken LH, Clarke SP, Sloane DM, Sochalski J, Silber JH (2002) Hospital nurse staffing and patient mortality, nurse burnout, and job dissatisfaction. JAMA 288 1987-1993.

5. National Quality Forum (2013) Quality positioning system. Accessed on May 6, 2013.

6. Wunderlich GS, Sloan F, Davis CK (1996) Nursing Staff in Hospitals and Nursing Homes: Is it adequate? The National Academies Press, Washington, DC.

7. Hartz AJ, Krakauer H, Kuhn EM, Young M, Jacobsen SJ, et al. (1989) Hospital characteristics and mortality rates. N Engl J Med 321: 1720-1725.

8. Shortell SM, Hughes EF (1988) The effects of regulation, competition, and ownership on mortality rates among hospital inpatients. N Engl J Med 318: 1100-1107.

9. Mitchell PH, Shortell SM (1997) Adverse outcomes and variations in organization of care delivery. Med Care 35: NS19-32.

10. Moses LE, Mosteller F (1968) Institutional differences in Postoperative death rates. Commentary on some of the findings of the National Halothane Study. JAMA 203: 492-494.

11. Pronovost PJ, Jenckes MW, Dorman T, Garrett E, Breslow MJ, et al. (1999) Organizational characteristics of intensive care units related to outcomes of abdominal aortic surgery. JAMA 281: 1310-1317.

12. Harless DW, Mark BA (2010) Nurse staffing and quality of care with direct measurement of inpatient staffing. Med Care 48: 659-663.

13. Needleman J, Buerhaus P, Mattke S, Stewart M, Zelevinsky K (2002) Nursestaffing levels and the quality of care in hospitals. N Engl J Med 346: 17151722.

14. Mark BA, Harless DW, McCue M, Xu Y (2004) A longitudinal examination of hospital registered nurse staffing and quality of care. Health Serv Res 39: 279300.

15. Sochalski J, Konetzka RT, Zhu J, Volpp K (2008) Will mandated minimum nurse staffing ratios lead to better patient outcomes? Med Care 46: 606-613

16. Kovner C, Gergen PJ (1998) Nurse staffing levels and adverse events following surgery in U.S. hospitals. Image J Nurs Sch 30: 315-321.

17. Lichtig LK, Knauf RA, Milholland DK (1999) Some impacts of nursing on acute care hospital outcomes. J Nurs Adm 29: 25-33.

18. Lankshear AJ, Sheldon TA, Maynard A (2005) Nurse staffing and healthcare outcomes: a systematic review of the international research evidence. ANS Adv Nurs Sci 28: 163-174.

19. Kane RL, Shamliyan TA, Mueller C, Duval S, Wilt TJ (2007) The association of registered nurse staffing levels and patient outcomes: systematic review and meta-analysis. Med Care 45: 1195-1204.

20. Blegen MA, Goode CJ, Spetz J, Vaughn T, Park SH (2011) Nurse staffing effects on patient outcomes: safety-net and non-safety-net hospitals. Med Care 49: 406-414

21. Manojlovich M, Sidani S, Covell CL, Antonakos CL (2011) Nurse dose: linking staffing variables to adverse patient outcomes. Nurs Res 60: 214-220.

22. Clarke SP (2007) Registered nurse staffing and patient outcomes in acute care: looking back, pushing forward. Med Care 45: 1126-1128.

23. Harless DW, Mark BA (2006) Addressing measurement error bias in nurse staffing research. Health Serv Res 41: 2006-2024.

24. Stone PW, Mooney-Kane C, Larson EL, Horan T, Glance LG, et al. (2007) Nurse working conditions and patient safety outcomes. Med Care 45: 571-578.
25. McGahan M, Kucharski G, Coyer F; Winner ACCCN Best Nursing Review Paper 2011 sponsored by Elsevier (2012) Nurse staffing levels and the incidence of mortality and morbidity in the adult intensive care unit: a literature review. Aust Crit Care 25: 64-77.

26. Numata Y, Schulzer M, van der Wal R, Globerman J, Semeniuk P, et al. (2006) Nurse staffing levels and hospital mortality in critical care settings: literature review and meta-analysis. J Adv Nurs 55: 435-448.

27. West E, Mays N, Rafferty AM, Rowan K, Sanderson C (2009) Nursing resources and patient outcomes in intensive care: a systematic review of the literature. Int J Nurs Stud 46: 993-1011.

28. Marsteller JA, Sexton JB, Hsu YJ, Hsiao CJ, Holzmueller CG, et al. (2012) A multicenter, phased, cluster-randomized controlled trial to reduce central lineassociated bloodstream infections in intensive care units*. Crit Care Med 40: 2933-2939.

29. Donabedian A (1966) Evaluating the quality of medical care. Milbank Mem Fund Q 44: 166-206

30. Hanrahan NP (2007) Measuring inpatient psychiatric environments psychometric properties of the Practice Environment Scale-Nursing Work Index (PES-NWI). Int J Psychiatr Nurs Res 12: 1521-1528.

31. Sexton JB, Helmreich RL, Neilands TB, Rowan K, Vella K, et al. (2006) The Safety Attitudes Questionnaire: psychometric properties, benchmarking data and emerging research. BMC Health Serv Res 6: 44

32. Greene WH (2012) Econometric Analysis. (7th edn), Prentice Hall, Upper Saddle River, NJ.

33. Stone PW, Pogorzelska M, Kunches L, Hirschhorn LR (2008) Hospital staffing and health care-associated infections: a systematic review of the literature. Clin Infect Dis 47: 937-944.

34. Donaldson N, Bolton LB, Aydin C, Brown D, Elashoff JD, et al. (2005) Impact of California's licensed nurse-patient ratios on unit-level nurse staffing and patient outcomes. Policy Polit Nurs Pract 6: 198-210.

35. Shekelle PG (2013) Nurse-patient ratios as a patient safety strategy: a systematic review. Ann Intern Med 158: 404-409.

36. Sales A, Sharp N, Li YF, Lowy E, Greiner G, et al. (2008) The association between nursing factors and patient mortality in the Veterans Health Administration: the view from the nursing unit level. Med Care 46: 938-945.

37. Jones CB (2005) The costs of nurse turnover, part 2: application of the Nursing Turnover Cost Calculation Methodology. J Nurs Adm 35: 41-49.

38. Hayes LJ, O'Brien-Pallas L, Duffield C, Shamian J, Buchan J, et al. (2006) Nurse turnover: a literature review. Int J Nurs Stud 43: 237-263. 